\title{
Rapid magnetic resonance of the lumbar spine was not better than radiographs for evaluating low back pain
}

Jarvik JG, Hollingworth W, Martin B, et al. Rapid magnetic resonance imaging vs radiographs for patients with low back pain: a randomized controlled trial. JAMA 2003;289:2810-8.

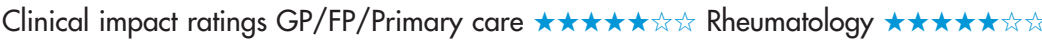

In patients with low back pain (LBP), are there clinical or economic consequences when replacing lumbar spine radiography (LSR) with rapid magnetic resonance imaging (MRI)?

METHODS

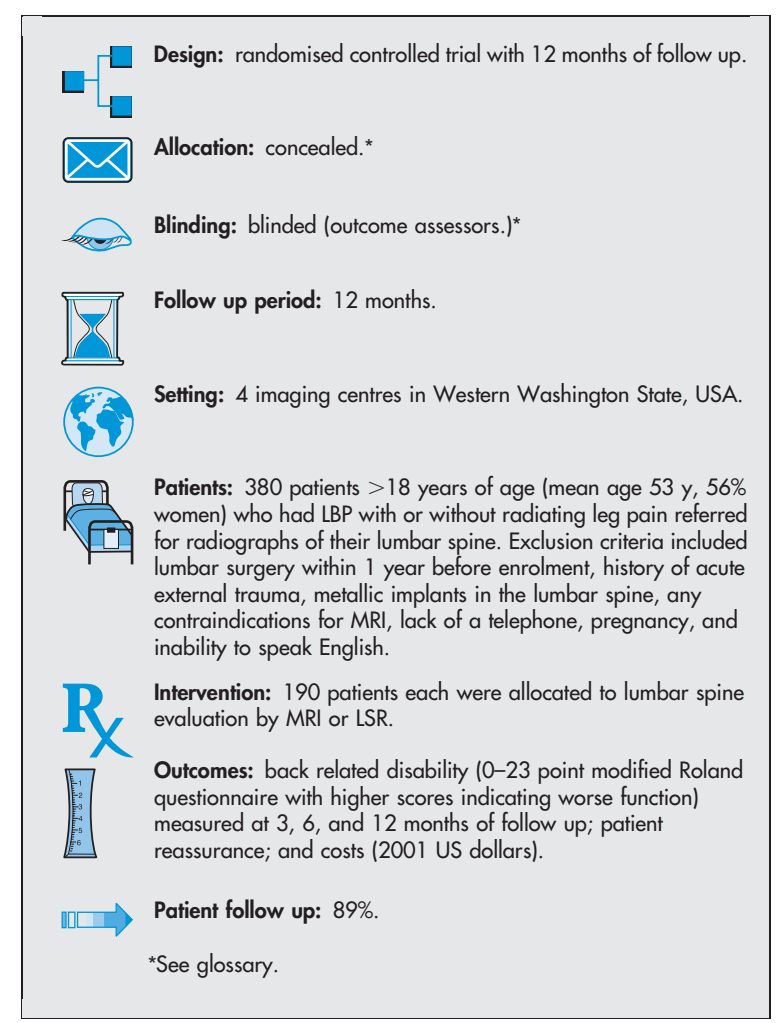

MAIN RESULTS

At 12 months, the groups did not differ for back related disability (table). The study had $80 \%$ power to detect a difference of 2 points on

For correspondence: Dr J G Jarvik, University of Washington, Seattle, WA USA. jarvikj@u.washington.edu

Sources of funding: Agency for Healthcare Research and Quality and National Institute for Arthritis and Musculoskeletal and Skin Diseases. the modified Roland Scale. However, patient reassurance was greater in the MRI group than in the LSR group (table). The groups did not differ for total societal costs (table).

\section{CONCLUSION}

In patients with low back pain, replacing lumbar spine radiography with rapid magnetic resonance imaging did not have clinical or economic consequences.

Commentary

M $\mathrm{RI}$ is an attractive alternative to LSR for investigating LBP. I provides more diagnostic information and avoids unnecessary radiation exposure. The study by Jarvik et al is the first to compare these 2 approaches in a randomised controlled trial of patients attending a variety of physicians working in primary and secondary care.

A possible limitation of the study is that only patients referred for LSR were included. These results might not be generalisable to those referred directly for MRI by their physicians. Additionally, the use of spinal imaging varies internationally so these results, especially the cost findings, might not be generalisable to other healthcare systems.

The groups did not differ for back related disability. The limits of the $95 \% \mathrm{Cl}$ for the difference in the modified Roland score were less than the pre defined smallest clinically important difference of 2. Although this study was not designed as an equivalence study, this narrow confidence interval suggests that clinicians could refer their patients for either MRI or conventional LSR without affecting clinical outcome. It is also noteworthy that patients randomised to MRI were substantially more reassured by the test. However, this study was not large enough to draw firm conclusions about other important outcomes, such as cost. The tendency for higher costs in the MRI group may reflect additional interventions for abnormalities identified by MRI. Overall, this study does not support replacing LSR with MRI for patients with simple back pain. MRI does not improve clinical outcome and may be more expensive. It also might result in more patients having surgical procedures.

However, it is not clear whether patients with simple back pain benefit from any imaging procedures. Other studies suggest that LSR does not improve outcome. ' In this study, no participants in either group had malignancy or infection. Based on data from this study, routine use of MRI for patients with simple LBP cannot be recommended. It did not improve clinical outcome, may increase costs, and did not identify any occult serious pathology.

Martin Underwood Institute of Community Health Sciences, Bart's and The London London, United Kingdom 1 Kendrick D, Fielding K, Bentley E, et al. Radiography of the lumbar spine in primary care patients with low back pain: randomised controlled trial. BMJ $2001 ; 322: 400-5$

Lumbar spine evaluation by rapid magnetic resonance imaging (MRI) v radiographs in low back pain at 12 weeks*

\begin{tabular}{llll}
\hline Outcomes & Rapid MRI & Radiograph & Difference (95\% Cl) \\
\hline Back pain related disability (mean MRS score) & 9.3 & 8.8 & $0.59(-0.87$ to 1.70$) \dagger$ \\
Patient reassurance (mean of 5 point scale) & 3.2 & 2.5 & $0.68(0.35$ to 0.99$) \dagger \ddagger$ \\
Total societal costs/patient (2001 US dollars) & 2380 & 2059 & $321(-458$ to 1100$)$ \\
\hline
\end{tabular}

${ }^{*}$ MRS $=$ 0-23 modified Roland Scale (higher score indicates worse health).

$\mathrm{TCl}$ (defined in glossary) from analysis of covariance adjusted for baseline score and study site.

¥Significant difference favours rapid MRI (higher score indicates greater reassurance). 